\title{
DYNAMICS OF COLLAPSE OF A CONFINED BOSE GAS
}

\author{
L.P. Pitaevskii ${ }^{1,2}$ \\ 'Department of physics, Technion, 32000 Haifa, Israel \\ ${ }^{2}$ Kapitza Institute for Physical Problems, 117454 Moscow, Russia
}

\begin{abstract}
Rigorous results on the nonlinear dynamics of a dilute Bose gas in an harmonic magnetic trap are presented and sufficient conditions for the collapse of the system are formulated. By using the virial theorem for the Gross-Pitaevskii equation in an external field we analyze the temporal evolution of the mean square radius $I=\int r^{2}|\psi|^{2} d V$ of the gas cloud. In the 2D case this quantity undergoes harmonic oscillations with frequency $2 \omega_{0}$. For a negative scattering length and for a negative value of the energy of the system, the gas cloud will collapse after a finite time interval. In the 3D case the system also collapses after a finite time for a state with negative energy. A stringent condition for the collapse is also derived.
\end{abstract}

\section{Extended Summary}

In $1925 \mathrm{~A}$. Einstien published a paper devoted to the generalization of Bose's work on the statistical mechanics of photons to an ideal gas of atoms. In this paper he predicted a remarkable phenomenon - the condensation of the atoms in a single quantum state. Such a condensation takes place below some critical temperature, which depends on the density of the gas. Below this temperature the number of the atoms $N_{0}$, which occupy the lowest quantum state $\mathbf{p}=0$, is proportional to the total number of atoms $N$, i.e. is macroscopically large:

$$
N_{0}=N\left[1-\left(T / T_{c}\right)^{3 / 2}\right] \text {. }
$$

The remaining atoms are distributed in the momentum space according to the usual Bose distribution with chemical potential $\mu=0$. The recent observation of Bose-Einstein condensation of atoms of ${ }^{87} \mathrm{Rb}[1],{ }^{23} \mathrm{Na}$ [2] and ${ }^{7} \mathrm{Li}$ [3] confined in magnetic traps has opened a new important field of investigation of quantum phenomena. In particular, a new feature of these systems is that they are significantly inhomogeneous so one can directly observe effects of quantum uncertainty on a macroscopical scale. The formal evidence of such a situation is that dynamics of these non-uniform gases at low temperatures can be properly described by a nonlinear equation for the "condensate wave function" $\psi(\mathbf{r}, t)[4],[5]$ :

$$
i \hbar \frac{\partial}{\partial t} \psi(\mathbf{r}, t)=\left(-\frac{\hbar^{2} \nabla^{2}}{2 m}+U(\mathbf{r})+g|\psi(\mathbf{r}, t)|^{2}\right) \psi(\mathbf{r}, t) .
$$


Here $U$ is the confining potential and $g=\frac{4 \pi \hbar^{2} a}{m}$, where $a$ is the s-wave scattering length. This equation is quite classical in its merit but contains explicitly the Plank's constant $\hbar$. (We will put $\hbar=m=1$ below.)

The physical situation is very different for ${ }^{87} \mathrm{Rb},{ }^{23} \mathrm{Na}$ on one side and ${ }^{7} \mathrm{Li}$ on the other. In the first case $a>0$ and the homogeneous state of the gas is stable. For ${ }^{7} \mathrm{Li}$ the scattering length $a$ is negative, which corresponds to an effective attraction, and the gas possesses negative compressibility. The uniform state in this case is unstable and the gas can undergo a "collapse" which is an unrestricted contraction. Fetter however has recently shown [7], using a variational approach, first employed by Baym and Pethick [6], that a system of a finite number $N$ of atoms in a trap can be in a metastable state for $N$ below some critical number $N_{\mathrm{c}}$.

In this paper we will present some rigorous results relative to the behaviour of the solution of Eq. (2). [8] We will analyze the temporal evolution of the quantity $I=\int r^{2}|\psi|^{2} d V$, which is proportional to the average square of the radius of the gas cloud.

Repeating differentiation of $I$ with respect to $t$, excluding $\partial \psi / \partial t$ from (2) and integrating by parts one gets for the two-dimensional motion of the gas in an isotropic cylindrical trap with a confining potential $U=\frac{\omega_{s}^{2} r^{2}}{2}, r^{2}=x^{2}+y^{2}$ :

$$
\frac{d^{2} I}{d t^{2}}=-4 \omega_{0}^{2} I+4 E, I=A \cos \left(2 \omega_{0} t+\gamma\right)+E / \omega_{3}^{2},
$$

where $E$ is the total energy of the gas. Eq. (3) describes harmonic pulsations of $I$ with constant frequency $2 \omega_{0}$ in spite of the nonlinear character of Eq. (2). Furthermore, one can see that for a negative value of the energy the positive quantity I approaches zero after a finite time smaller than $\pi / 2 \omega_{0}$. This means complete contraction of the cloud.

Results for the 3D case are not so conclusive. Consider an anisotropic harmonic trap with a confining potential of the form $U=\frac{\alpha_{i k} x_{i} x_{k}}{2}$. One gets:

$$
\frac{d^{2} I}{d t^{2}}=6 E-5 \int \alpha_{i k} x_{i} x_{k} d V-2 K
$$

where $K=\frac{\mathrm{i}}{2} \int|\nabla \psi|^{2} d V$ is the kinetic energy. Eq. (4) gives the inequality:

$$
\frac{d^{2} I}{d t^{2}}+5 \omega_{m}^{2} I-6 E<0
$$


where $\omega_{m}^{2}$ is the smallest eigenvalue of the $\alpha_{i k}$ matrix. We can prove again that the system collapses after a finite time for a negative $E$.

One can get more rigorous sufficient conditions of the collapse using the method developed in [9]-[10]. In this method an important part is played by two inequalities imposed on the kinetic energy:

$$
I K \geq \frac{9}{8} N^{2}, \frac{1}{2} \int|\psi|^{4} d V \leq \beta N^{1 / 2} K^{3 / 2}, \beta=0.0575
$$

Combining the inequalities, one immediately gets an inequality for the energy $E$ as a function of $K$ :

$$
E(K)>K+\frac{9}{16} \omega_{m}^{2} N^{2} K^{-1}-\beta|g| K^{3 / 2} N^{1 / 2}
$$

Introducing dimensionless variables $\varepsilon$ and $\kappa$ defined by $E=\frac{3}{4} N \omega_{m} \varepsilon, K=\frac{3}{4} N \omega_{m} \kappa$, we can rewrite this equation as:

$$
\varepsilon>\kappa+\kappa^{-1}-1.18 \frac{2}{3} \sigma \equiv f(\kappa, \sigma)
$$

where $\sigma=\sqrt{2 / \pi} N|a| \omega_{m}^{1 / 2}$.

Behaviour of the function $f(\kappa)$ depends on the value of $\sigma$. For $\sigma \geq \sigma_{c}=0.454$ the curve is a monotonous one. Let the point $\varepsilon^{*}, \kappa^{*}$ to be an intersection of the curve $\varepsilon=f(\kappa)$ and the straight line $\varepsilon=\kappa / 3$. Let us be

$$
E<E^{*}=\frac{3}{4} N \omega_{m} \varepsilon^{*}>0
$$

Then the kinetic energy $K>K^{*}=3 E^{*}$ for any $t$. This means that equation (4) leads to the inequality $\frac{d^{2} I}{d t^{2}}+5 \omega_{m}^{2} I-6\left(E-E^{*}\right)<0$. This formula obviously demonstrates that at condition (9) we get the collapse.

If $\sigma<\sigma_{c}$, the curve $\varepsilon=f(\kappa)$ has this case a minimum $\varepsilon_{m}$ and a maximum $\varepsilon_{M}$. Let us consider an initial state, where

$$
\varepsilon_{m}<\varepsilon<\varepsilon_{M}, \kappa_{1}<\kappa<\kappa_{2}
$$


Since any solution of (2) evolves continuously, the kinetic energy $\kappa$ will stay in the same range at every time. The uncertainty relation (6) gives then that $I>\frac{3 N}{2 \omega_{m} \kappa_{2}}$. Thus the mean square radius stays restricted and collapse of the cloud as a whole is impossible.

If $E<E^{*}$ the cloud will collapse as in the previous case. The evolution of the cloud for $E>E^{*}$ and $K$ out the interval (10) demands additional investigation.

I believe that suggested method can be useful for investigation of developing instabilities in different unstable media.

\section{References}

[1] M.N. Anderson, J.R. Ensher, M. R. Matthews, C.E. Wieman, and E.A. Cornell, Science 269, 198 (1995).

[2] K.B. Davis, M.O. Mewes, M.R. Andrews, N.J. Van Druten, D.S. Durfee, D.M. Kurn, and W. Ketterly, Phys. Lett. 75, 3969 (1995).

[3] C.C. Bradley, C.A. Sackett, and R.G. Hulet, Phys. Rev. Lett. 78, 985 (1997).

[4] E.P Gross, Nuovo Cimento 20, 454 (1961).

[5] L.P. Pitaevskii, Sov. Phys. JETP 13, 451 (1961).

[6] G. Baym and C. Pethick, Phys. Rev. Lett. 76, 6 (1996).

[7] A. Fetter, Journ. Low Temp. Phys. 106, 643 (1997).

[8] L.P. Pitaevskii Physics Letters A 221, 14 (1996).

[9] E.A. Kuznetov, A.M. Rubenchik, and V.E. Zakharov, Phys. Rep., 142, 103 (1986).

[10] S.K. Turitsyn, Phys. Rev. E 47, R13 (1993). 